\title{
Properties of nearby giant star-forming regions
}

\author{
Deidre A. Hunter \\ Lowell Observatory, \\ 1400 West Mars Hill Road, Flagstaff, AZ 86001, USA
}

\begin{abstract}
I review the properties of two nearby giant $\mathrm{H}$ II regions -30 Doradus and NGC 604, and of two nearby young star complexes now past the H II region phase - Constellation III and NGC 206. I discuss the stellar populations, mode of star formation, gas content, and kinematics as clues to what conditions may be like in more distant starburst environments.
\end{abstract}

\section{Introduction}

I have been asked to review the properties of some nearby giant $\mathrm{H}$ II regions with the view to what they tell us about starburst environments. These giant starforming complexes are places to test assumptions and deductions from global properties of more distant regions of intense star formation, and in so far as conditions in distant galaxies are similiar, they can show us what is going on in more distant places. In this review I will focus on 30 Doradus in the LMC, as the quintessential giant $\mathrm{H}$ II region, and NGC 604 in M 33. I will also include two young regions that are not $\mathrm{H}$ II regions because they are old enough to have blown the gas away. These star complexes, Constellation III in the LMC and NGC 206 in M 31, are examples of what giant H II regions evolve to. This raises one cautionary note: properties of giant star-forming complexes change rapidly over timescales of $10 \mathrm{Myr}$ as the region evolves. Therefore, for some properties we are only capturing the region at one particular instant of time: 30 Doradus and NGC 604 represent the properties of mature giant H II regions while NGC 206 and Constellation III are post-H II complexes.

\section{Some global properties}

A few basic global properties of the four regions are catalogued in Table 1. One can see that the star-forming complexes are of order $1 \mathrm{kpc}$ in size. The nebulae themselves are of order $400 \mathrm{pc}$ and have emission equal to that of nearly a thousand Orion nebulae. The original gas clouds that formed these complexes were of order $10^{7} \mathrm{M}_{\odot}$. These regions are bright in the UV; NGC 206 stands out as one of the brightest regions in M 31 in UV images. They are also bright in X-rays, and have complex kinematics until they blow a hole in the gas. The holes that Constellation III and NGC 206 sit in are of order $1 \mathrm{kpc}$ in diameter.

Efremov (1995) has suggested that large-scale complexes of OB associations are a common mode of star formation in disk galaxies, and that they are physical entities rather than chance collections. He argues that the star complex is the 
largest and initial scale of star formation that begins with gas superclouds of order $10^{7} \mathrm{M}_{\odot}$. A top-down scenario of star formation leads to individual giant molecular clouds within this region that form individual $\mathrm{OB}$ associations and clusters over an extended period of time. For the most part, the regions that we are focussing on here are the star complexes that Efremov has identified, with the possible exception of NGC 604 which is smaller.

Star formation within a star complex is not instantaneous. From 30 Doradus, Constellation III, and NGC 206 we see that the bulk of the star formation proceeds over a time scale of order $10 \mathrm{Myr}$. Furthermore, in 30 Doradus (Lortet \& Testor 1991) and Constellation III (Reid et al. 1987; Olsen et al. 1997; Braun et al. 1997; Dolphin \& Hunter 1998) there is no correlation of age with distance from the center of the region, indicating that star formation has not proceeded in any obvious sequential fashion; there may be some star induced star formation within the complexes but it is not so orderly as to be a function of radius.

Table 1. Some global properties

\begin{tabular}{|c|c|c|c|c|}
\hline & 30 Doradus $^{a}$ & NGC $604^{b}$ & NGC $206^{c}$ & Constellation $\mathrm{III}^{d}$ \\
\hline $\begin{array}{l}\text { distance }(\mathrm{pc}) \\
\text { age }(\mathrm{Myr})^{e} \\
\text { diameter }(\mathrm{pc})\end{array}$ & $\begin{array}{c}0.055 \\
\leq 10(20) \\
\text { nebula } \sim 10 \\
\text { overall } \sim 1000\end{array}$ & $\begin{array}{c}0.84 \\
4 \\
\text { nebula } \sim 420\end{array}$ & $\begin{array}{c}0.77 \\
3-8(40-50) \\
\text { stars } \sim 950\end{array}$ & $\begin{array}{c}0.05 \\
9-16 \\
\text { stars } \sim 900\end{array}$ \\
\hline$L_{\mathrm{H} \alpha, 0}\left(10^{39} \mathrm{erg} \mathrm{s}^{-1}\right)^{f}$ & 18 & 5 & 0 & 0 \\
\hline$M_{\mathrm{H} \text { I }}\left(10^{6} \mathrm{M}_{\odot}\right)^{g}$ & 14 & 2 & 2 & 15 \\
\hline$M_{\mathrm{H}_{2}}\left(10^{6} \mathrm{M}_{\odot}\right)$ & 4 & 4 & 0 & 0 \\
\hline $\begin{array}{l}M_{\text {stars }}\left(10^{5} \mathrm{M}_{\odot}\right)^{h} \\
\text { WR stars? }\end{array}$ & $\geq 3(110)$ & $\begin{array}{l}2 \\
Y\end{array}$ & $\begin{array}{l}4 \\
Y\end{array}$ & 24 \\
\hline X-ray $\left(10^{6} \mathrm{~K}\right)$ & $\begin{array}{c}Y \\
2-10\end{array}$ & $\begin{array}{l}Y \\
1\end{array}$ & $\begin{array}{l}Y \\
\ldots\end{array}$ & $\begin{array}{l}N \\
2\end{array}$ \\
\hline 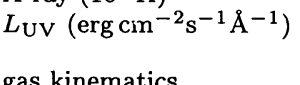 & $\ldots$ & $\ldots$ & $\begin{array}{c}F(2000) \simeq 3 \times 10^{-13} \\
F(1550) \simeq 0.5 \times 10^{-13}\end{array}$ & $\ldots$ \\
\hline gas kinematics & complex & complex & gas hole & gas hole \\
\hline
\end{tabular}

${ }^{a}$ Kennicutt (1984), Walborn (1991), McGee \& Milton (1966), Cohen et al. (1988), Massey \& Hunter (1998), McGregor \& Hyland (1981), Hyland et al. (1992), Rubio et al. (1992), Wang \& Helfand (1991a), Walborn \& Blades (1997), Lortet \& Testor (1991), Rubio et al. (1998).

${ }^{b}$ Kennicutt (1984), Hunter et al. (1996a), Rosa \& D'Odorico (1982), Drissen et al. (1993), Wilson \& Scoville (1992), Wright (1971).

${ }^{c}$ van den Bergh (1966), Brinks (1981), Chernin et al. (1995), Hunter et al. (1996b), Massey \& Johnson 1998, Carruthers et al. (1978), Deharveng et al. (1980), Hill et al. (1992).

${ }^{d}$ Braun et al. (1997), Olsen et al. (1997), Dolphin \& Hunter (1998), Dopita et al. (1985), Bomans et al. (1994).

${ }^{e}$ Values in parenthesis show older ages that there is some evidence for in the region.

${ }^{f}$ Units of about 100 Orion nebulae.

${ }^{g}$ Values for Constellation III and NGC 206 are estimates based on the H I content of the surrounding shell.

${ }^{h}$ Extrapolating the stellar IMF 0.1 to $120 \mathrm{M}_{\odot}$. NGC 206: includes a factor of 1.5 to correct for cluster missed by the HST-WFPC2 census. 30 Doradus: Value is for the region surveyed by Parker (1993) plus R136; value in parenthesis extrapolates Parker region to entire 30 Doradus complex. Con III: extrapolates Dolphin \& Hunter (1998) survey to entire complex. 
Efremov has suggested that it is from these $10^{7} \mathrm{M}_{\odot}$ 'superclouds' that the giant molecular clouds condense. 30 Doradus and NGC 604 are still young enough for us to get some sense of the molecular clouds out of which the stars have formed. Both regions indeed contain multiple clouds comparable to the giant molecular clouds in our Galaxy. In 30 Doradus, there are two clouds $420-500$ pc in diameter with masses of $4.5-7 \times 10^{6} \mathrm{M}_{\odot}$ (resolution of $140 \mathrm{pc}$ and $1.3 \mathrm{~km} \mathrm{~s}^{-1}$, Cohen et al. 1988). In NGC 604, four clouds have been identified with diameters of $40-64 \mathrm{pc}$ and masses of $0.3-0.5 \times 10^{6} \mathrm{M}_{\odot}$ (resolutions of $30 \mathrm{pc}$ and $2.6 \mathrm{~km} \mathrm{~s}^{-1}$, Viallefond et al. 1992, Wilson \& Scoville 1992).

\section{Stellar populations}

Starbursts and regions of intense high mass star formation have been said to be deficient in lower mass stars or to have unusual stellar initial mass functions (IMFs). For example, in M 82 Rieke et al. (1993) have argued from global properties that the lower mass limit $M_{l}$ is no less than a few $\mathrm{M}_{\odot}$; similarly observational arguments have been made that Mrk 171 and Tol 65 have made only OB-type stars in their recent starburst (Augarder \& Lequeux 1985); and theoretical arguments in the past have suggested that $M_{l}$ could be as high as $10 \mathrm{M}_{\odot}$ in regions of intense massive star formation (Silk 1977, 1995; Elmegreen \& Lada 1977; Larson 1985; Elmegreen 1997). However, observational arguments are necessarily based on global properties. In nearby star complexes, at least with $H S T$, we can do a direct stellar census far enough down in mass to be able to place constraints on $M_{l}$. So, let us look at what we know about the stellar populations of these four complexes.

\subsection{Massive stars}

The massive star content is summarized for the four regions in Table 2. All four regions contain hundreds of massive stars. In 30 Doradus and NGC 206 most of the massive stars are found in $\mathrm{OB}$ associations scattered throughout the star complex; in NGC 604 they are found in two major groupings of stars; in Constellation III many of the more massive stars have died off by now.

However, 30 Doradus is unusual among these four complexes in containing the compact, luminous star cluster R 136. This star cluster is probably what a small globular cluster was like when it was young. $\mathrm{R} 136$ has a half-light radius of $1.7 \mathrm{pc}$, a mass (extrapolated from 2.8 to $0.1 \mathrm{M}_{\odot}$ ) of $6 \times 10^{4} \mathrm{M}_{\odot}$, and an $\mathrm{M}_{V}=-11$ (Hunter et al. 1996c). Within the cluster there are 39 O3-type stars that have been observed and its most massive stars reach masses of $150 \mathrm{M}_{\odot}$ (Massey \& Hunter 1998). And all this within a few parsecs! Therefore, R 136 epitomizes the intense star-forming environment. The stellar content of this very young cluster - the massive stars are only 1-2 Myr old - is normal (see next section); it contains so many very massive stars only because it is so young and so especially rich in stars altogether. Massey \& Hunter (1998) have suggested, in fact, that the upper stellar mass limit that has been observed so far, $\sim 150 \mathrm{M}_{\odot}$, is only statistical. Although there must be some physical limit, we have been limited in observing the mass ceiling by the small numbers of stars formed as well as the older age of regions. 
Table 2. Massive star contents

\begin{tabular}{|c|c|c|}
\hline 30 Doradus $^{a}$ & NGC $604^{b}$ & NGC $206^{c}$ \\
\hline R $136 \quad$ Parker region & & \\
\hline
\end{tabular}

\begin{tabular}{lcccc}
\hline $\mathrm{N}\left(\mathrm{M}_{V} \leq-4\right)$ & 120 & 450 & 186 & $280:$ \\
$\mathrm{N}(\mathrm{WC})$ & $(1)$ & 3 & 14 candidates & 2 \\
$\mathrm{~N}(\mathrm{WN})$ & 4 & 8 & & 2 \\
$\mathrm{~N}(\mathrm{WR}) / \mathrm{N}\left(\mathrm{M}_{V} \leq-4\right)$ & 0.03 & 0.02 & $\leq 0.08$ & 0.01 \\
$\mathrm{~N}(\mathrm{WC}) / \mathrm{N}(\mathrm{WN})$ & $(0.25)$ & 0.38 & $\ldots$ & 1.0 \\
\hline
\end{tabular}

a'Parker region' includes only the region surveyed by Parker (1993) outside of R 136; R 136 is from Hunter et al. (1996c). The WC in the R 136 region is probably an interloper and not part of the cluster itself. The WN number does not include O3If*/WN6-A stars: 6 in R 136, 2 in 30 Doradus.

${ }^{b}$ Hunter et al. (1996a), Drissen et al. (1993).

${ }^{c}$ Hunter et al. (1996b), a factor of 1.5 has been included to account for the part of the cluster not surveyed with HST-WFPC2; Massey \& Johnson (1998).

Wolf-Rayet stars are also present in all regions except Constellation III which is too old by now to contain any. I have entered some survey numbers in Table 2 that are of interest to people who study Wolf-Rayet galaxies. The WC/WN ratio is a function of metallicity (see Figure 8 of Massey \& Johnson 1998) and the ratios in the table are what are expected for the metallicities of the galaxies. The $\mathrm{N}(\mathrm{WR}) / \mathrm{N}\left(M_{V} \leq-4\right)$ ratios are also roughly what one expects from few Myr old populations (Leitherer \& Heckman 1995).

However, R 136 offers a cautionary tale. Although R 136 contains four WolfRayet stars, none of them are evolved objects as one usually expects Wolf-Rayet stars to be. They are over-luminous - by a factor of 10 - hydrogen-burning stars that are 1-2 Myr old rather than $\geq 3$ Myr (de Koter et al. 1997; Massey $\&$ Hunter 1998). Furthermore, the equivalent widths of the $\lambda 4686$ line is 70 $100 \AA$, which is about normal for a WN star. Therefore, the flux in $\lambda 4686$ is about 10 times higher than for a normal WN star, and these hydrogen-burning WN stars will each masquerade as $10 \mathrm{WN}$ stars if you are counting stars from an integrated $\lambda 4686$ flux. Thus, these stars could be partly responsible for the unusually high WN/O star ratio that is inferred in Wolf-Rayet galaxies.

\subsection{Stellar Initial Mass Function}

Slopes $\Gamma$ of the stellar IMFs measured in these regions are listed in Table 3. The IMF, as a measure of the proportion of stars formed as a function of the mass of the star, tells us what the stellar populations of the star complexes are. Slopes comparable to that of Salpeter are also seen in most other clusters and associations in these and other galaxies. We see, therefore, that IMFs of stellar associations and clusters are 'normal' (that is, like Salpeter), to within the uncertainties, wherever one measures it, even in R 136! Abnormal IMFs have only been measured for field massive stars (Massey et al. 1995b). At this meeting, we have heard that global properties lead one to infer that interacting spirals have IMFs that are steeper than Salpeter and have unusually low upper mass limits (see Joseph, these Proceedings) and that Wolf-Rayet galaxies have 
Table 3. Stellar Initial Mass Functions

\begin{tabular}{lccl}
\hline region & $\Gamma$ & $\begin{array}{c}\text { mass range } \\
\left(\mathrm{M}_{\odot}\right)\end{array}$ & reference \\
\hline R 136 & $-1.3 \pm 0.1$ & $2.8-120$ & Hunter et al. 1996c, Massey \& Hunter 1998 \\
30 Doradus & $-1.5 \pm 0.2$ & $\geq 12$ & Parker \& Garmany 1993 \\
NGC 604 & $-1.6 \pm 0.7$ & $6.5-18$ & Hunter et al. 1996a \\
NGC 206 & $-1.4 \pm 0.5$ & $6-15$ & Hunter et al. 1996b \\
Constellation III & -0.9 to $-2.1 \pm 0.2$ & $6.5-15$ & Dolphin \& Hunter 1998 \\
\hline
\end{tabular}

shallower than Salpeter IMFs (see Contini et al., these Proceedings). We need to understand the discrepancy between these studies and those of individual star surveys of nearby star-forming regions.

\subsection{Lower stellar mass limits}

$\mathrm{R} 136$ is the most extreme environment in which to test claims that the lower stellar mass limit should be high. It is also the place among our four complexes with the most stringent observed limit to $M_{l}$. In R $136 M_{l} \leq 2.8 \mathrm{M}_{\odot}$ (Hunter et al. $1996 \mathrm{c}$ ). There are stars measured to $\sim 1 \mathrm{M}_{\odot}$ in HST-WFPC2 images, but photometric and detection uncertainties are too large to say anything significant below $\sim 2.8 \mathrm{M}_{\odot}$. HST-NICMOS observations in progress will, however, address the lower mass stars. For the time being, we can say that in an intense region of star formation like R 136, there are stars in normal proportions down at least to a few $\mathrm{M}_{\odot}$, not just to $10-20 \mathrm{M}_{\odot}$.

\section{Mode of star formation}

In this section I want to examine the mode of star formation in these star complexes. By that I mean the richness, or number of stars formed, and the concentration, or number of stars per unit area that have formed in the region. In other words what are the star-forming regions like? Obviously, R 136 is the most extreme in terms of both of these parameters. It contains many stars and they are crammed into a small space. In fact, the concentration of stars in R 136 is about 300 times what is found in typical OB associations. Richness and concentration are shown for a variety of regions in Figure 1 (Parker \& Garmany 1993; Massey et al. 1995a,b; Dolphin \& Hunter 1998; Hunter et al. 1996a,b,c,1997; Hunter \& Thronson 1995; Lynds et al. 1998). From this figure one can see that some, maybe even most, giant $\mathrm{H}$ II regions are just scaled $\mathrm{OB}$ associations; they have formed more stars but at the same spatial concentration as in typical OB associations. This includes 30 Doradus outside R 136, the bulk of the star formation in Constellation III, and NGC 604. NGC 206 has formed more stars than R 136 but is unusual in having a fairly low spatial concentration. Constellation III has formed some knots that are comparable in concentration, although not richness, to the populous cluster NGC 1818, but these knots still are less concentrated and rich than R 136 by factors of ten. Here we can also compare with three more distant galaxies. The giant northern $\mathrm{H}$ II region in the 


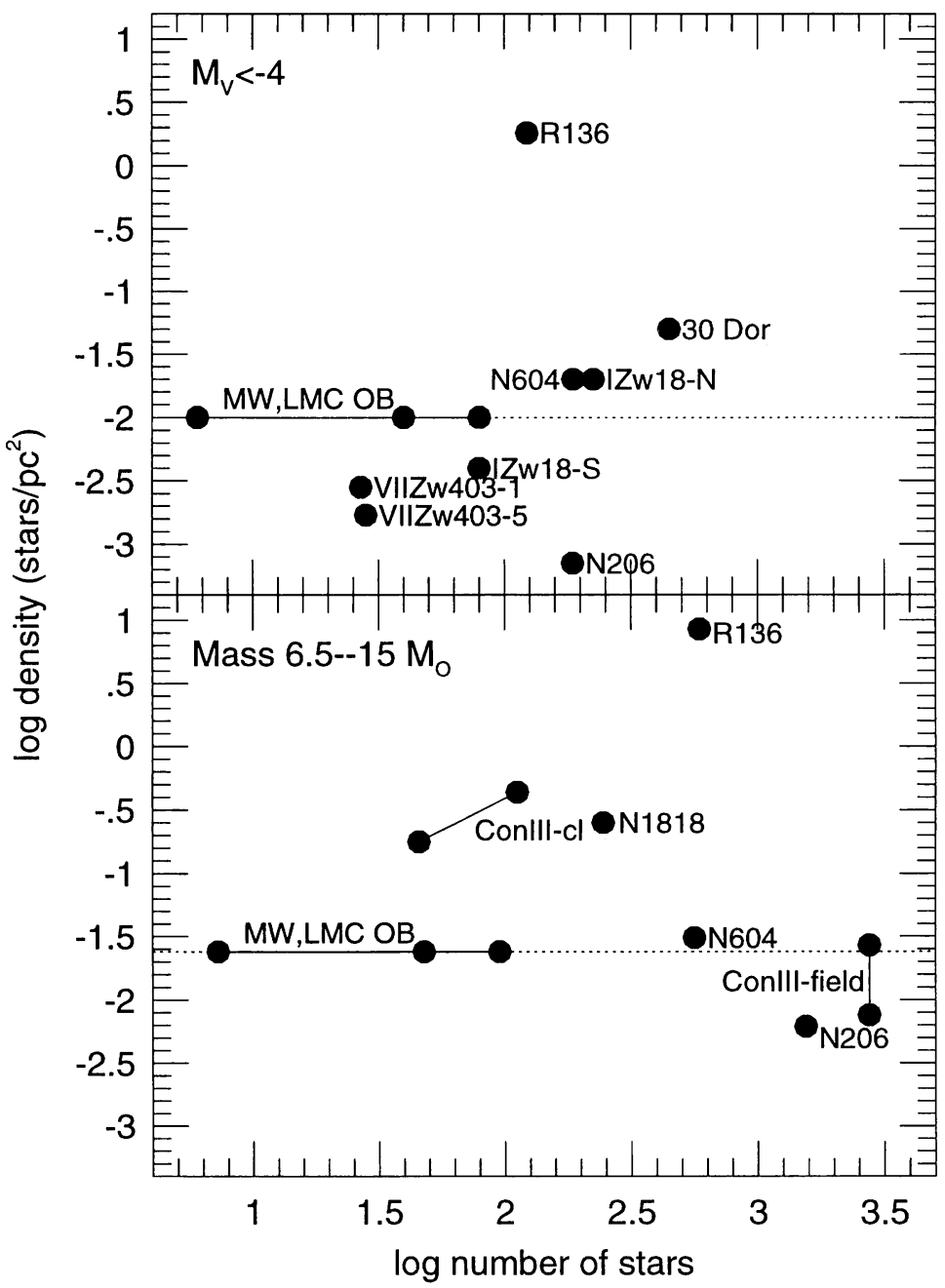

Figure 1. Richness and concentration of stars formed in various starforming regions. The top panel is for stars brighter than -4 , approximately all O-type stars, a parameter that varies as the region evolves. The bottom panel is for stars with masses $6.5-15 \mathrm{M}_{\odot}$, a quantity that does not vary for regions as young as these but that is harder to measure in more distant galaxies.

Blue Compact Dwarf galaxy I Zw 18, the southern H II region in that galaxy, the clusters in VII Zw 403, and the diffuse star formation in the Local Group starburst galaxy IC 10 (see poster by Hunter, these Proceedings) are all typical or 
scaled versions of $\mathrm{OB}$ associations. Therefore, not all BCDs/starbursts contain unusual star-forming regions even if they contain giant $\mathrm{H}$ II regions.

However, at least one, 30 Doradus, does contain a superstar cluster, R 136 . In this regard, the 30 Doradus complex and Constellation III offer an interesting comparison. Both complexes contain or contained similar amounts of gas, are about the same size, formed stars over comparable time periods, and formed scattered $\mathrm{OB}$ associations. In many ways these two star complexes are very similar. Yet, 30 Doradus formed R 136 and Constellation III only formed little knots that are 30 times less concentrated and 10 times less rich in stars. Why? Is it that the distribution of the gas within the region was different? Is it the influence of the bar potential on 30 Doradus? This is an outstanding question in understanding star formation processes.

\section{The H II regions}

30 Doradus has been extensively studied as an $\mathrm{H}$ II region (Elliott et al. 1977, Scowen et al. 1998, Meaburn 1984, Kennicutt \& Chu 1994, Chu \& Kennicutt 1994, Cohen et al. 1988). We see that the H II region sits on one side of a tongue of HI. Many of the ionized gas filaments are ionization fronts eating into neutral gas (see schematic model by Elliot et al. 1977, Figure 28). The kinematics are complex with multiple velocity components resulting from 10 50 pc sheets, 3-15 pc shells with expansion velocities up to $200 \mathrm{~km} \mathrm{~s}^{-1}$, and larger shells up to $100 \mathrm{pc}$ with velocities of $35 \mathrm{~km} \mathrm{~s}^{-1}$. The general turbulent velocity is $\sim 30-40 \mathrm{~km} \mathrm{~s}^{-1}$. All of this is due to supernova remnants that have gone off throughout the region and the stellar winds, especially those from the stars in $R$ 136. The kinetic energy in the ionized gas is estimated to be $10^{53}$ erg for the entire nebula over $10^{7}$ years. In addition to all of this activity in the ionized gas, there is an expanding $\mathrm{CO}$ ring with a diameter of $500 \mathrm{pc}$, a mass of $3.8 \times 10^{6} \mathrm{M}_{\odot}$, an expansion velocity of $17 \mathrm{~km} \mathrm{~s}^{-1}$, and kinetic energy of $3 \times 10^{52} \mathrm{erg}$.

NGC 604 has been described as similar to the 30 Doradus nebula (Rosa \& Solf 1984, Sabalisck et al. 1995, Yang et al. 1996, Rosa \& D'Odorico 1982). It has supersonic motions of order $40 \mathrm{~km} \mathrm{~s}^{-1}$ due to thermal broadening, stellar winds, supernova remnants, and virial motion. There are shells and sheets with velocities up to $100 \mathrm{~km} \mathrm{~s}^{-1}$. NGC 604 is also located on the edge of a huge $\mathrm{HI}$ cloud and is what is termed a 'blister' $\mathrm{H}$ II region. It too is churned up by supernovae, strong stellar winds, as well as the blister expansion. The kinetic energy in the $\mathrm{H}$ II is estimated to be $10^{52} \mathrm{erg}$.

In X-rays 30 Doradus and Constellation III have been well studied (Wang \& Helfand 1991a,b; Bomans et al. 1994; review by Wang 1998). 30 Doradus exhibits X-ray bubbles surrounded by $\mathrm{H} \alpha$ shells plus diffuse X-ray emission. The gas shows a range in temperatures of $2-10 \mathrm{MK}$ with the high energy gas mostly found around R 136 . The hot gas contains $10^{52.5} \mathrm{erg}$ and $10^{4} \mathrm{M}_{\odot}$. This hot gas is the result of stellar winds and supernovae. The hot gas and H II gas are mixing in the core around $\mathrm{R} 136$. The cavity has evaporative flows resulting from pressure gradient between the ionization fronts and interior of the cavity. Wang has a spectacular color image of 30 Doradus, combining X-rays, $\mathrm{H} \alpha$, and $\mathrm{UV}$, on his web page and I would urge you to look at it to appreciate the real complexity of this region. 
Constellation III represents an older region, now a supershell (LMC-4). It exhibits diffuse X-ray emission with ridges of higher surface brightness, a temperature of several MK, and an X-ray luminosity for $0.1-2.4 \mathrm{keV}$ of $10^{37} \mathrm{erg} \mathrm{s}^{-1}$.

\section{What happens next?}

We know that the concentration of massive stars in Constellation III has blown a hole in the neutral gas, creating a supershell around it. An interesting question is whether this region, and regions like it, actually break out of the galactic disk. This has important consequences for the evolution of the galaxy. It is already fairly amazing that the LMC-4 supershell is $1.2 \mathrm{kpc}$ in diameter. That seems extraordinarily large. Yet, Bomans et al. (1994) argue that the hot gas in LMC-4 has not yet even broken through to the halo and that, because of overpressure, it is still expanding into the ambient medium. However, the data are partial and noisy, and Dopita et al. (these Proceedings) argue that the Xrays in Constellation III are leaking in from the $\mathrm{H}$ II regions in the surrounding supershell. On the other hand, the LMC-2 supershell, adjacent to 30 Doradus, is argued by Wang \& Helfand (1991b) from, admittedly noisy, Einstein data to have broken out with only a diameter of $500 \mathrm{pc}$, leading to a scale height estimate for the LMC that is smaller than that of the Milky Way. However, Points et al. (1996) argue that the structures in LMC-2 are more complicated than those of an expanding shell. Thus, in these two cases - supershells LMC-4 and LMC-2 - it is not entirely clear whether break-out has or will occur. We can expect that 30 Doradus and NGC 604, like Constellation III and NGC 206, will blow substantial holes in the gaseous disk, but what happens beyond that is still unclear. Again, we find contradictions in interpretation of observations: Dopita et al. (these Proceedings) argue that many of the shells in the LMC have already broken out while Brinks \& Walter (these Proceedings) argue that the $\mathrm{HI}$ disks of irregulars are too thick to allow breakout. This is another area needing further study to iron out the contradictions.

Another issue is whether subsequent generations of stars beyond the complexes will form as a direct consequence of the energy input from these complexes. In the case of Constellation III we can see that a modest second generation has formed; they are the H II regions in the $\mathrm{H}$ I shell surrounding the Constellation (Dopita et al. 1985). On the other hand, theoretical considerations by Silk (1997) of the interstellar medium porosity suggest that numerous holes in the interstellar medium of galaxies make it even more difficult for galaxies to form stars overall. That this is a real issue is driven home by the stunning H I map of the LMC produced by Kim et al. (1998). These maps show the surroundings of Constellation III to contain many holes. Constellation III may well provide an example of the limitations of this process: There has been a modest second generation, but will there be a significant third generation? The H I map of Kim et al. suggests that the interstellar medium may be too fragmentary to support a significant third generation beyond the complex. 


\section{Summary}

These star complexes are found to be both simple and complicated. They are simple in that the stellar populations are, for the most part, assemblages of smaller building blocks that we are familiar with and contain stars in proportions that we have come to expect. They are complex in the interplay of massive stars with the leftover gas.

Acknowledgments. I wish to thank HST for grants GO-06417.02-95A and GO-07370.02-96A that have funded part of the $\mathrm{R} 136$ research and Bart Wakker for insights into scale heights.

\section{References}

Augarder, R., Lequeux, J. 1985, A\&A 147, 273

Bomans, D.J., Dennerl, K., Kürster, M. 1994, A\&A 283, L21

Braun, J.M., Bomans, D.J., Will, J.M., De Boer, K.S. 1997, A\&A 328, 167

Brinks, E. 1981, A\&A 95, L1

Carruthers, G.R., Opal, C.B., Heckathorn, H.M. 1978, ApJ 225, 346

Chernin, A.D., Efremov, Y.N., \& Voinovich, P.A. 1995, MNRAS 275, 313

Chu, Y.-H., Kennicutt, R.C. 1994, ApJ 425, 720

Cohen, R.S., Dame, T.M., Garay, G., Montani, J., Rubio, M., Thaddeus, P. 1988, ApJ 331, L95

de Koter, A., Heap, S.R., Hubeny, I. 1997, ApJ 477, 792

Deharveng, J.M., Milliard, B., Laget, M., Jakobsen, P. 1980, A\&A 88, 52

Dolphin, A.E., Hunter, D.A. 1998, AJ 116, 1275

Dopita, M.A., Mathewson, D.S., Ford, V.L. 1985, ApJ 297, 599

Drissen, L., Moffat, A.F.J., Shara, M.M. 1993, AJ 105, 1400

Elliott, K.H., Goudis, C., Meaburn, J., Tebbutt, N.J. 1977, A\&A 55, 187

Efremov, Y.N. 1995, AJ 110, 2757

Elmegreen, B.G. 1997, ApJ 486, 944

Elmegreen, B.G., Lada, C.J. 1977, ApJ 214, 725

Hill, J.K., et al. 1992, ApJ 395, L33

Hunter, D.A., Baum, W.A., O’Neil, E.J., Lynds, R. 1996a, ApJ 456, 174 (NGC 604)

Hunter, D.A., Baum, W.A., O’Neil, E.J., Lynds, R. 1996b, ApJ 468, 633 (NGC 206)

Hunter, D.A., Light, R.M., Holtzman, J.A., Lynds, R., O’Neil, E.J., Grillmair, C.J. 1997, ApJ 478, 124

Hunter, D.A., O'Neil, E.J., Lynds, R., Shaya, E., Groth, E., Holtzman, J. 1996c, ApJ 459, L27 (R 136)

Hunter, D.A., Thronson, H.A. 1995, ApJ 452238

Hyland, A.R., Straw, S., Jones, T.J., Gatley, I. 1992, MNRAS 257, 391

Kennicutt, R.C. 1984, ApJ 287, 116

Kennicutt, R.C., Chu, Y.-H. 1994, in: G. Tenorio-Tagle (ed.), Violent Star Formation, From 30 Doradus to QSOs (Cambridge: CUP), p. 1

Kim, S., Staveley-Smith, L., Dopita, M.A., Freeman, K.C., Sault, R.J., Kesteven, M.J., McConnell, D. 1998, ApJ 503, 674

Larson, R.B. 1985, MNRAS 214, 379 
Leitherer, C., Heckman, T.M. 1995, ApJS 96, 9

Lortet, M.-C., Testor, G. 1991, A\&AS 89, 185

Lynds, R., Tolstoy, E., O'Neil, E.J., Hunter, D.A. 1998, AJ 116, 146

Massey, P., Johnson, K., Degioia-Eastwood, K. 1995a, ApJ 454, 151

Massey, P., Lang, C.C., Degioia-Eastwood, K., Garmany, C.D. 1995b, ApJ 438, 188

Massey, P., Hunter, D.A. 1998, ApJ 493, 180

Massey, P., Johnson, O. 1998, ApJ 505,793

McGee, R.X., Milton, J.A. 1966, Australian J Phys 19, 343

McGregor, P.J., Hyland, A.R. 1981, ApJ 250, 116

Meaburn, J. 1984, MNRAS 211, 521

Olsen, K.A.G., Hodge, P.W., Wilcots, E.M., Pastwick, L. 1997, ApJ 475, 545

Parker, J.Wm. 1993, AJ 106, 560

Parker, J.Wm., Garmany, C.D. 1993, AJ 106, 1471

Points, S.D., Chu, Y.-H., Bomans, D.J., Smith, R.C. 1996, BAAS 28, 924

Reid, N., Mould, J., Thompson, I. 1987, ApJ 323, 433

Rieke, G.H., Loken, K., Rieke, M.J., Tamblyn, P. 1993, ApJ 412, 99

Rosa, M., D'Odorico, S. 1982, A\&A 108, 339

Rosa, M., Solf, J. 1984, A\&A 130, 29

Rubio, M., Barbá, R.H., Walborn, N.R., Probst, R.G., García, J., Roth, M.R. 1998, AJ 116,1708

Rubio, M., Roth, M., Garcia, J. 1992, A\&A 261, L29

Sabalisck, N.S.P., Tenorio-Tagle, G., Castañeda, H.O., Muñoz-Tuñón, C. 1995, ApJ 444,200

Scowen, P.A., Hester, J.J., Sankrit, R., Callagher, J.S. et al. 1998, AJ 116, 163

Silk, J. 1977, ApJ 214, 718

Silk, J. 1995, ApJ 438, L41

Silk, J. 1997, ApJ 481, 703

van den Bergh, S. 1966, AJ 71, 219

Viallefond, F., Boulanger, F., Cox, P., Lequeux, J., Pérault, M., Vogel, S.N. 1992, A\&A 265,437

Walborn, N.R. 1991, in: R. Haynes \& D. Milne (eds.), The Magellanic Clouds, Proc. IAU Symp. No. 148 (Dordrecht: Kluwer), p. 145

Walborn, N.R., Baldes, J.C. 1997, ApJS 112, 457

Wang, Q. 1998, in: T. Richtler \& J.M. Braun (eds.), The Magellanic Clouds and Other Dwarf Galaxies, Proc. Bonn/Bochum-Graduiertenkolleg Workshop (Shaker Verlag), p...

Wang, Q., Helfand, D.J. 1991a, ApJ 370, 541

Wang, Q., Helfand, D.J. 1991b, ApJ 379, 327

Wilson, C.D., Scoville, N. 1992, ApJ 385, 512

Wright, M.C.H. 1971, Ap. Letters 7, 209

Yang, H., Chu, Y.-H., Skillman, E.D., Terlevich, R. 1996, AJ 112, 146

\section{Discussion}

Selman: In your work for the high-mass range (Massey \& Hunter 1998) you find an IMF slope, which is indistinguishable from Salpeter. For the intermediate-mass range 
(Hunter et al. 1996) you find a slope only marginally flatter, and conclude that the two segments are consistent with Salpeter. But in Massey \& Hunter (1998) you use the best fit IMF, which is flatter, to predict the number of stars with $M>50 \mathrm{M}_{\odot}$ and conclude that the prediction agrees with the numbers observed. Nevertheless, had you used the Salpeter slope, you would have been short by a factor of 2.5. Could you comment?

Hunter: When we take the intermediate-mass stars $\left(\Gamma=-1.0 \pm 0.1,2.8-15 \mathrm{M}_{\odot}\right)$ and predict the numbers of high mass stars $\left(50-120 \mathrm{M}_{\odot}\right)$, we predict numbers that are close to what we observe. When we formally fit the high-mass stars only, we get a slope of $\Gamma=-1.3 \pm 0.1$. So what this says is that there is not much statistical difference between $\Gamma=-1.3$ and $\Gamma=-1.0$ for the high-mass stars only. Perhaps we should quote $\Gamma=-1.0$ for the whole range rather than $\Gamma=-1.3$, but in either case $\Gamma$ is comparable to Salpeter within the uncertainties. The $\Gamma$ is simply not precise enough to pin it down to more than a few 0.1 . What we were particularly trying to show by extrapolating the intermediate-mass IMF up and predicting the number of high-mass stars was to show that the IMF was continuous and that there was nothing extraordinary in the large number of high-mass stars that we observed.

Marston: There appear to be no RSG or LBV stars in dense star clusters. Is there any evidence for the restriction of the slow outflows from such objects in regions of high pressure from stellar winds? And might this affect their evolution?

Hunter: I will have to difer that question to a stellar expert in the audience.

Elena Terlevich: We have detected one RSG and one star in the process of becoming an LBV in NGC 604 (spectroscopy): E. Terlevich et al. (1996 MNRAS 279, 1219), confirmed by poster of Maíz-Apellániz et al. (these Proceedings) with HST colours.

Polcaro: In the figures of the WR/O stars ratios you gave, did you include only the 'real' WR stars (i.e., the chemically evolved high-mass stars), or also what was called in the previous talk the 'main sequence' WR?

Hunter: In Table 1 I include all WR stars. If one restricts oneself to only evolved WR stars, the only number that would change is that for R 136 which would go to zero. But, one of the points I tried to make was that in fact the presence of 'main sequence' WR stars will complicate the interpretation of $f(\lambda 4686)$ in distant galaxies, partly because they are significantly younger than normal WR stars, and partly because they are so much more luminous in $\lambda 4686$.

Polcaro: Comment on the reply: Actually, if the so-called MS-WR are included, these ratios do not give any information about the stellar evolution of the stellar association. 